\title{
Video Article \\ Imaging of HIV-1 Envelope-induced Virological Synapse and Signaling on Synthetic Lipid Bilayers
}

\author{
Kathleen C. Prins ${ }^{\star^{1,2}}$, Gaia Vasiliver-Shamis ${ }^{\star 2,3}$, Michael Cammer ${ }^{1,2}$, David Depoil ${ }^{1,2}$, Michael L. Dustin ${ }^{2}$, Catarina E. Hioe ${ }^{1,4}$ \\ ${ }^{1}$ Department of Pathology, New York University Langone School of Medicine \\ ${ }^{2}$ Program in Molecular Pathogenesis, Marty and Helen Kimmel Center for Biology and Medicine and Skirball Institute for Biomolecular Medicine \\ ${ }^{3}$ Laboratory of Molecular Immunogenetics, National Institute of Arthritis and Musculoskeletal and Skin Diseases, National Institutes of Health \\ ${ }^{4}$ Veteran Affairs New York Harbor Healthcare System \\ * These authors contributed equally
}

URL: https://www.jove.com/video/3757

DOI: doi:10.3791/3757

Keywords: Immunology, Issue 61, TIRF microscopy, planar bilayer, HIV envelope, virological synapse

Date Published: 3/8/2012

Citation: Prins, K.C., Vasiliver-Shamis, G., Cammer, M., Depoil, D., Dustin, M.L., Hioe, C.E. Imaging of HIV-1 Envelope-induced Virological Synapse and Signaling on Synthetic Lipid Bilayers. J. Vis. Exp. (61), e3757, doi:10.3791/3757 (2012).

\section{Abstract}

Human immunodeficiency virus type 1 (HIV-1) infection occurs most efficiently via cell to cell transmission ${ }^{2,10,11}$. This cell to cell transfer between $\mathrm{CD}^{+} \mathrm{T}$ cells involves the formation of a virological synapse (VS), which is an F-actin-dependent cell-cell junction formed upon the engagement of HIV-1 envelope gp120 on the infected cell with CD4 and the chemokine receptor (CKR) CCR5 or CXCR4 on the target cell ${ }^{8}$. In addition to gp120 and its receptors, other membrane proteins, particularly the adhesion molecule LFA-1 and its ligands, the ICAM family, play a major role in VS formation and virus transmission as they are present on the surface of virus-infected donor cells and target cells, as well as on the envelope of HIV-1 virions $s^{1,4,5,6,7,13}$. VS formation is also accompanied by intracellular signaling events that are transduced as a result of gp120engagement of its receptors. Indeed, we have recently showed that $\mathrm{CD} 4^{+} \mathrm{T}$ cell interaction with gp120 induces recruitment and phosphorylation of signaling molecules associated with the TCR signalosome including Lck, CD3ろ, ZAP70, LAT, SLP-76, Itk, and PLCY ${ }^{15}$.

In this article, we present a method to visualize supramolecular arrangement and membrane-proximal signaling events taking place during VS formation. We take advantage of the glass-supported planar bi-layer system as a reductionist model to represent the surface of HIV-infected cells bearing the viral envelope gp120 and the cellular adhesion molecule ICAM-1. The protocol describes general procedures for monitoring HIV-1 gp120-induced VS assembly and signal activation events that include i) bi-layer preparation and assembly in a flow cell, ii) injection of cells and immunofluorescence staining to detect intracellular signaling molecules on cells interacting with HIV-1 gp120 and ICAM-1 on bi-layers, iii) image acquisition by TIRF microscopy, and iv) data analysis. This system generates high-resolution images of VS interface beyond that achieved with the conventional cell-cell system as it allows detection of distinct clusters of individual molecular components of VS along with specific signaling molecules recruited to these sub-domains.

\section{Video Link}

The video component of this article can be found at https://www.jove.com/video/3757/

Protocol

\section{Labeling GP120}

Fluorescent dyes used in this protocol are effective for binding to proteins, are sufficiently photo-stabile for microscopy imaging, and match the excitation wavelengths of the lasers available with our microscope. These three criteria need to be met when selecting fluorescent molecules for tagging proteins.

1. Exchange $\mathrm{His}_{6}$-tagged gp120 DH12 (a gift from Dr. Michael Cho, lowa State University) protein buffer to sterile PBS using a centrifugal filter unit (30 kDa MWCO). Make sure gp120 concentration is not more than $1 \mathrm{mg} / \mathrm{ml}$. Note: His $_{6}$-tagged gp120 proteins from other X4 or R5 tropic strains have also been tested and are now available commercially from Immune Technologies.

2. Add sodium bicarbonate $\mathrm{pH} 9.0$ to your protein solution to obtain $50 \mathrm{mM}$ final concentration of sodium bicarbonate with $\sim \mathrm{pH} 8.5$.

3. Add amine reactive Alexa Fluor 488 (AF488) fluorescent dye, which is dissolved in water at a 10 -fold molar excess. Incubate this mixture for 1-2 hours at room temperature in the dark.

4. To remove the excess dye, use the centrifugal filter unit as before and add fresh sterile PBS to the column. Repeat this step until all free dye is removed (3-4 washes with $4 \mathrm{ml}$ PBS). Spin down until gp120 is concentrated to about $1 \mathrm{mg} / \mathrm{ml}$. Note: Removing free dye by dialysis or centrifugal filter unit only works if the dye is water soluble, or else gel filtration can be used.

5. To measure the fluorescence intensity per molecule of protein (F/P) of the labeled protein, determine the concentrations (in $\mu M$ ) of the fluorescent dye at the appropriate wavelength (for example, at $488 \mathrm{~nm}$ for AlexaFluor 488) and protein (we use a NanoDrop spectrophotometer using the 'proteins and labels' setting) and then divide these two numbers giving you the F/P ratio. The same procedure 
is used for other proteins and dyes such as ICAM-1 and Cy5. Note: Other types of spectrophotometers can be used to obtain the protein concentration and fluorescent dye concentration to obtain the F/P ratio if a NanoDrop is not available.

\section{Flow Cell Assembly and Bilayer Preparation}

The general procedure for flow cell and bilayer preparation has been described in detail previously ${ }^{14}$. Here we outline specifically the protocol to prepare bilayers containing $\mathrm{His}_{6}$-tagged gp120 DH12 on a Bioptech flow cell. For studies involving infectious viruses or infected cells and when small volumes are necessary due to limited reagents, a disposable lbidi flow chamber (sticky Slide I ${ }^{0.2}$ Luer) may be used and followed with the same bi-layer preparation procedure in Steps 2.4-2.9. Information for Ibidi flow chambers can be obtained from the company website, http:// www.ibidi.com/service/display_material/CA_8p_EN_150dpi.pdf.

1. Prepare Pirhana solution ( $45 \mathrm{ml}$ sulfuric acid (Trace Metal Grade $98 \%$ ) and $15 \mathrm{ml} 30 \%$ hydrogen peroxide) ${ }^{14}$. With plastic clamps, immerse cover slips in the Pirhana solution for 15 minutes.

2. Transfer cover slips to a beaker filled with pica-purified water and wash each one under running water for 2 min (one minute on each side). Place on rack to dry.

3. Assemble Bioptechs FCSII chambers as previously described ${ }^{14}$.

4. A liposome mixture containing $12.5 \% \mathrm{Ni}^{2+}$ chelating lipids is used for capturing and presenting His $\mathrm{H}_{6}$-gp 120 on the bilayer surface ${ }^{16}$. Add $1 \mu \mathrm{ll}$ drops of liposome mixture onto the microaqueduct slide without touching the tip to the glass. To prepare the maximum of 5 bilayers per flow cell, repeat up to 4 more times so that there are five $1 \mu$ dots as shown previously ${ }^{14}$.

5. Place cover slip on top of lipids. Cover the white retaining ring with a stainless steel clamp base unit, flip the whole apparatus over and seal. Mark location of bilayers with a marker. Incubate for 10 mins at room temperature.

6. Attach tubing with two-way stopcock to left perfusion tube, as shown in video. Generate a positive meniscus at the end of the tubing with the 3-way stopcock on it (tubing has been previously filled with HEPES-buffered saline (HBS) containing human serum albumin (HSA) (HBS/ HSA:50 ml 10x HBS [10x HEPES buffered saline: $200 \mathrm{mM}$ HEPES, pH 7.2, $1.37 \mathrm{M} \mathrm{NaCl}, 50 \mathrm{mM} \mathrm{KCl,} 7 \mathrm{mM} \mathrm{Na}_{2} \mathrm{HPO}_{4}, 60 \mathrm{mM} \mathrm{D}^{-g l u c o s e}$ ] $20 \mathrm{ml} 25 \mathrm{x} \mathrm{HSA}, 500 \mu \mathrm{l} 1 \mathrm{M} \mathrm{CaCl}_{2}, 1 \mathrm{ml} 1 \mathrm{M} \mathrm{MgCl}_{2}$ and $\mathrm{dH}_{2} \mathrm{O}$ to bring a total volume to $500 \mathrm{ml}$ and filter with $0.22 \mu \mathrm{m}$ filter) and is devoid of bubbles. Connect tubing to right perfusion tube and gently push buffer through flow cell.

7. Block bilayer with $\sim 300 \mu \mathrm{l}$ of casein containing $100 \mu \mathrm{M} \mathrm{NiCl}_{2}$ by filling $1 \mathrm{ml}$ syringe and attaching to the open portion of 3-way stopcock. Gently push buffer through and close both stopcocks before disengaging the syringe. Incubate for 30 min at room temperature.

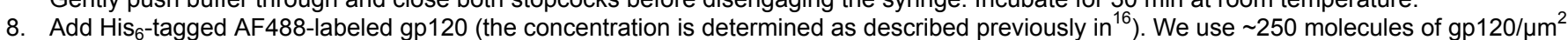
to mimic the local density of Env clusters found on HIV-1 virion surface ${ }^{17}$. Similarly, we use $\sim 250$ molecules/ $\mu \mathrm{m}^{2}$ of ICAM-1 on the bilayer as previously reported ${ }^{14}$. Load into flow cell as done with casein. Incubate for $30 \mathrm{~min}$ in dark (cover with foil) at room temperature. The same procedure is used to incorporate other proteins such as $\mathrm{His}_{12}$-tagged Cy5-labeled ICAM-1 onto the bilayer.

9. Wash bilayers with $5 \mathrm{ml}$ of buffer.

\section{Quality Control of Bilayers via FRAP}

Proteins in bilayers must be laterally diffusible. Before adding cells onto bilayers, it is important to assure the mobility of proteins in the prepared bilayer. Here we describe a Fluorescent Recovery After Photobleaching (FRAP) method ${ }^{3}$ that may be performed manually on most objective illuminated TIRF, wide field epifluorescence, or laser scanning confocal microscopes. The goal is to image fully uniform fields of fluorescence in the lipid bilayer, bleach a spot, and to monitor the return of unquenched molecules to the bleached area. A recovery of $50 \%$ in 2 minutes may be acceptable. All the major microscope manufacturers (Leica, Nikon, Olympus, Zeiss) sell objective illuminated TIRF systems that work well for this application. While each company provides variations of TIRF illumination and other operational features, the image quality is essentially the same.

1. Use a low excitation intensity to minimize bleaching. Use a high numerical aperture objective such as a N.A. 1.3 to $1.4540 x, 60 x$ or $100 x$. When using a standard fluorescent or TIRF microscope, to reduce the light intensity, put neutral density filters in the excitation light path. Most laser systems may be set for low illumination by setting an acusto optical tunable filter (AOTF) or acusto optical beam splitter (AOBS) with low voltage.

2. Record a "pre-bleach" image. If using a cooled CCD camera, to compensate for low light conditions binning may be set to $2 \times 2$ or $4 \times 4$, the gain may be set high, or long exposures (for instance on the order of seconds) may be used. If using a confocal, the pinhole aperture may be opened; the gain set high, and slow scanning or averaging used.

3. Bleach a spot. If using a standard fluorescence or TIRF microscope, remove all ND filters to allow for maximum intensity illumination, close the field diaphragm, and expose the sample for a few seconds. If using a laser scanning confocal, set the laser power maximum and zoom in approximately $4 x$ to $8 x$ to bleach a spot. A warning for confocal usage: do not zoom in too high because the bilayer may be damaged by excessively concentrated photons.

4. At ten to fifteen second intervals, record images using the same illumination and recording settings as in 3.1 and 3.2 . Within two to three minutes the spot should recover intensity approaching that of the "pre-bleach"image. Quick recovery is the sign of a successful mobile bilayer. If the spot remains dark, and especially if the edge retains high contrast, the fluorescent proteins are immobile in the bilayer and should not be used for the experiment.

Note: In our current microscope, we can deliver $0.9 \mathrm{~mW}$ of $641 \mathrm{~nm}$ light to a circular spot with an area of $240 \mu \mathrm{m}^{2}$ which bleaches fluorescent ICAM at typical concentrations in less than 4 seconds. Readers should find that critical alignment of an $\mathrm{Hg}$ or Xe lamp with bleaching times less than 30 seconds is more than sufficient to perform this assay in a repeatable manner. We would also refer you to reference ${ }^{3}$ for additional details.

\section{Injection of Cells and Immunofluorescence Staining}

1. Warm up flow cell to $37^{\circ} \mathrm{C}$ (this can be done in a $37^{\circ} \mathrm{C}$ incubator with no $\mathrm{CO}_{2}$ for about $30 \mathrm{~min}$ ). 
2. Add activated human $\mathrm{CD}^{+} \mathrm{T}$ cells $\left(2-5 \times 10^{6} /\right.$ flow cell) in $\mathrm{HBS} / \mathrm{HSA} \sim 400 \mu \mathrm{l}$ by $1 \mathrm{ml}$ syringe. Let cells attach to bilayer for $\sim 45 \mathrm{~min}$ in the $37^{\circ} \mathrm{C}$ incubator. If the Ibidi chambers are used, supply additional buffer every $15-20 \mathrm{~min}$.

3. Fix the cells by injecting $2 \%$ paraformaldehyde and incubate for $10 \mathrm{mins}$ at $37^{\circ} \mathrm{C}$.

4. Wash $3 x$ with $1 \mathrm{ml}$ room temperature PBS buffer. Permeabilize cells by injecting $0.1 \%$ Triton $X-100$ for 5 min at room temperature.

5. Wash $3 x$ with $1 \mathrm{ml}$ of room temperature PBS buffer (when probing for phosphorylated proteins, you can add sodium vanadate at $1 \mathrm{mM}$ final concentration or another phosphatase inhibitor in PBS to prevent phosphate removal during processing). Block using casein with $5 \%$ goat serum for 25 mins at room temperature. The type of animal serum depends on the secondary antibody.

6. Wash $3 x$ with $1 \mathrm{ml}$ of room temperature PBS buffer. Add primary antibody in $\sim 300 \mu \mathrm{l} \mathrm{buffer/flow}$ cell for $30 \mathrm{~min}-1 \mathrm{hr}$ at room temperature. To detect the initial membrane-proximal activation signal, we use antibodies specific for phosphorylated Lck (pLck), total Lck or Fyn.

7. Wash $3 x$ with $1 \mathrm{ml}$ of room temperature PBS buffer. Add appropriate fluorescently labeled secondary antibody in buffer as done with primary antibody. For our experiments, we use Alexa Fluor 568-labeled goat anti-rabbit secondary. Incubate for 20 min at room temperature. Wash $3 x$ with $1 \mathrm{ml}$ of PBS buffer.

8. Note: It is essential to have a control bilayer that is treated with only secondary antibody (no primary antibody). Follow steps 4.1-4.5 and skip 4.6, then proceed with step 4.7. This will determine the level of non-specific binding of your secondary antibody. Alternatively, one can use directly labeled primary antibodies.

\section{Image acquisition by TIRF microscopy}

TIRF microscopy allows excitation of fluorescent signals limited to a $250 \mathrm{~nm}$ or thinner plane at the glass substrate and cell interface. This guarantees image acquisition of only signals from the lipid bilayer and from the immediately apposed cell membranes. Therefore, only molecules at the synapse are imaged. Because TIRF images only the membrane-proximal area at the bottom of the cell, proteins that are internalized or redistributed into the dorsal membrane will not be detected. It may then be important to additionally acquire images by standard widefield or confocal microscopy to determine accumulation or distribution of the proteins at the synaptic area as compared to the other volumes of the cell.

All the major microscope manufacturers (Leica, Nikon, Olympus, Zeiss) sell objective illuminated TIRF systems that work well for this application. While each company provides variations of TIRF illumination and other operational features, the image quality is essentially the same. Each TIRF microscope has its own details for operation, but the following general rules apply to obtain high-resolution imaging.

- Illumination should be low to avoid bleaching.

- The camera should have a linear response.

- The camera should be capable of imaging a wide dynamic range with no saturation.

- All settings should be set constant for quantitative analyses.

\section{Data Analysis}

To study the intracellular signals activated as a result of $\mathrm{CD} 4^{+} \mathrm{T}$ cell interaction with gp120 at the VS, quantitative analyses of TIRF images are done to determine whether or not intracellular signaling molecules such as Lck and Fyn are activated and recruited to the synapse ${ }^{15}$. Here we describe a simple method applicable for most image analysis application packages such as ImageJ and Metamorph. We have used ImageJ, which runs on Windows, Macintosh, and Linux, for our method here ${ }^{12}$.

In the Analyze > Set Measurements tab, check the boxes for Area and Mean gray value. When you make a region of interest on the image that is a polygon or a freehand traced closed shape and do Analyze > Measure, the software will put the data from this measurement in a results table. The Area will be in number of pixels or in $\mu \mathrm{m}^{2}$. The Mean is the average intensity in arbitrary units. It must have background intensity subtracted from it. Multiplying the mean minus background by area returns the integrated intensity of the protein in arbitrary units.

1. Set the measurements to Mean and Area.

2. Open images for one experimental condition.

3. For each cell, trace and measure the region of interest (mean) and trace and measure a region outside the region of interest (background).

4. When done with one experimental condition, either copy measurements and paste into Excel or other spreadsheet program or save the measurements.

5. Return to step 6.2 for each experimental condition.

6. When measurements are completed, calculate the results. The formulas are: Average intensity = mean - background Integrated intensity $=$ Average intensity * area

\section{Representative Results}

To measure activation and recruitment of the initial membrane-proximal signaling molecules Lck and Fyn as a result of interaction with gp120 at the VS, primary human CD4 $4^{+}$T cells were introduced onto bilayers bearing gp120 and ICAM-1 ${ }^{15}$. Cells introduced to bilayers with only ICAM-1 served as a control to define the basal levels of signaling. Specific fluorescence intensity was measured within the gp120 contact area for cells on gp120 and ICAM-1 containing bilayers and within the whole contact area for cells interacting with the ICAM-1 bilayer. An increase of average fluorescence intensity is a sign of augmented recruitment and activation of the signaling molecule to the VS. This will be further demonstrated by a comparable increase in the integrated fluorescence intensity. If however, there is no change in the integrated fluorescence intensity, this indicates a redistribution of the signaling molecule.

After the cells interacted with bilayers containing gp120 and ICAM-1, total Lck and pLck(Y394) were recruited to the VS interface and colocalized with gp120 (Figs. 1 \& 2). The average intensity of total Lck (Fig. 1) was higher on the bilayer containing both gp120 and ICAM-1 than with ICAM-1 alone, but the integrated intensity levels (Fig. 1) were similar, suggesting that Lck is redistributed into a central cluster upon CD4 ${ }^{+} \mathrm{T}_{\text {cell }}$ 
binding to gp120. However, quantification of pLck(Y394) (Fig. 2) showed that the average intensity on gp120 and ICAM-1 containing bilayers was higher than that on ICAM-1 alone bilayers, and the integrated intensity was higher on bilayers with both gp120 and ICAM-1 than on ICAM-1 alone bilayers. This indicates that while the levels of total Lck at the interface are similar in cells adhering onto gp120 and ICAM-1 and ICAM-1 alone bilayers, gp120 binding increased phosphorylation of residue Y394 at the Lck activation loop. In contrast, Fyn was not recruited to the VS (Fig. 3), as more Fyn was present in the contact area of cells on ICAM-1 alone bilayers than on bilayers containing both gp120 and ICAM-1. Consequently, we conclude that Lck, not Fyn, is the active kinase in the HIV-1 gp120-induced VS.

Figures: Membrane-proximal signaling at HIV-1 gp120-induced VS. Images of representative cells on the gp120+ICAM-1 bilayer (top panels) and the ICAM-1 bilayer (bottom panels) are shown. Fluorescence intensities of the individual cells were quantified within manually traced areas of the cell footprints as illustrated by the region marked with the yellow line in Figure 1. Quantification of average and integrated intensities detected by TIRF microscopy are presented in the left and right graphs, respectively. A total of 30 to 350 cells were quantified for each condition. Bars $=5 \mu \mathrm{m}$. Data from one of three repeat experiments are shown

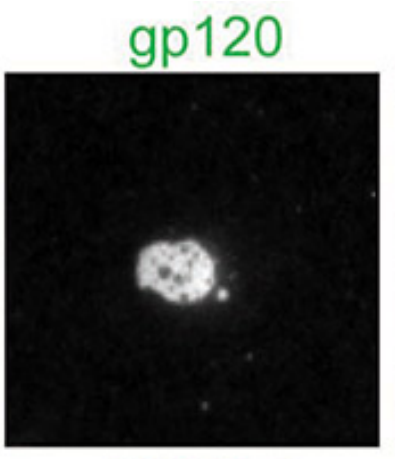

ICAM-1
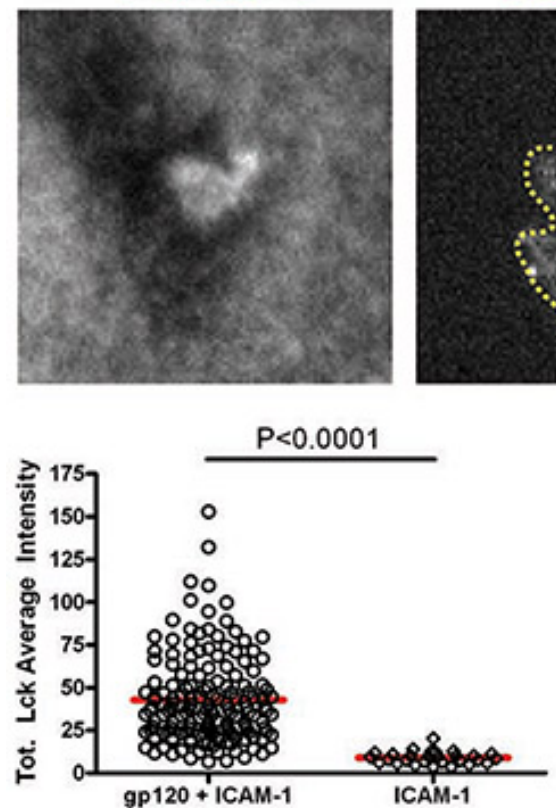

Total Lck

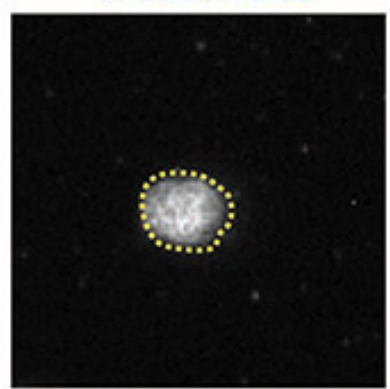

Total Lck
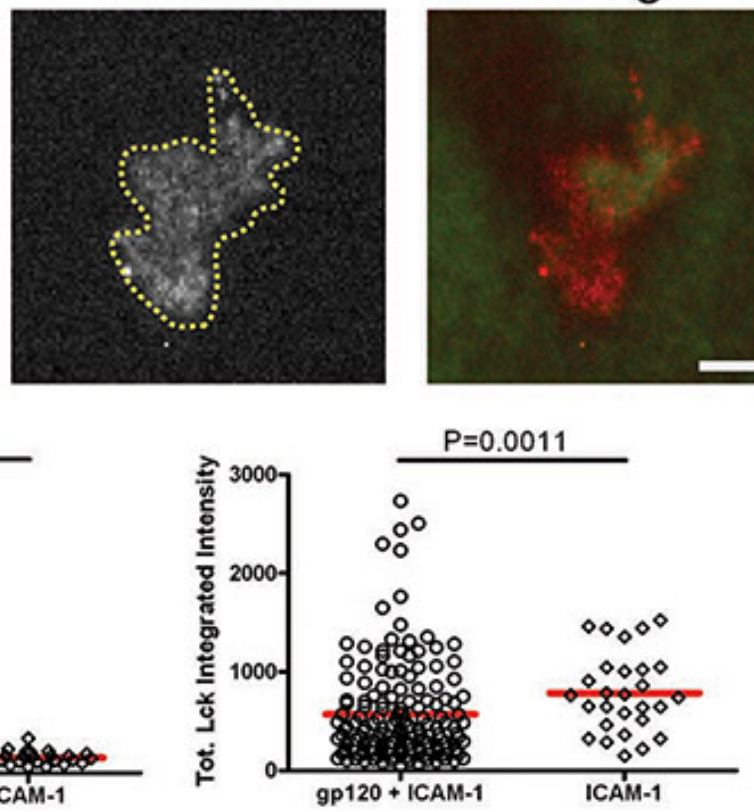

Figure 1. $\mathrm{CD}^{+} \mathrm{T}$ cells were introduced onto bilayers containing gp120 and ICAM-1 or ICAM-1 alone for 45 min and then fixed and stained for total Lck. 

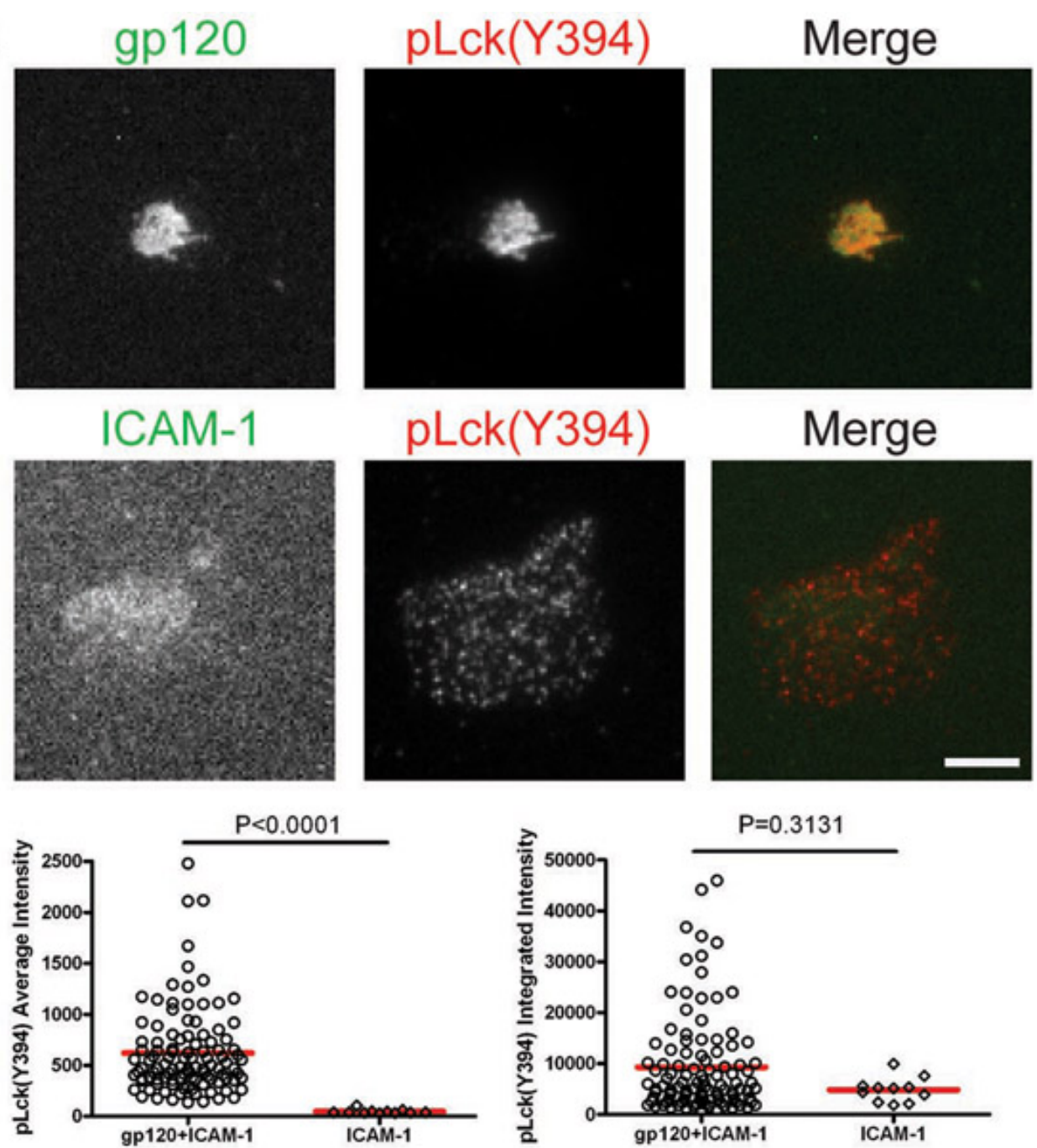

Figure 2. $\mathrm{CD}^{+} \mathrm{T}$ cells were introduced onto bilayers containing gp120 and ICAM-1 or ICAM- 1 alone for 45 min and then fixed and stained for pLck(Y394). 

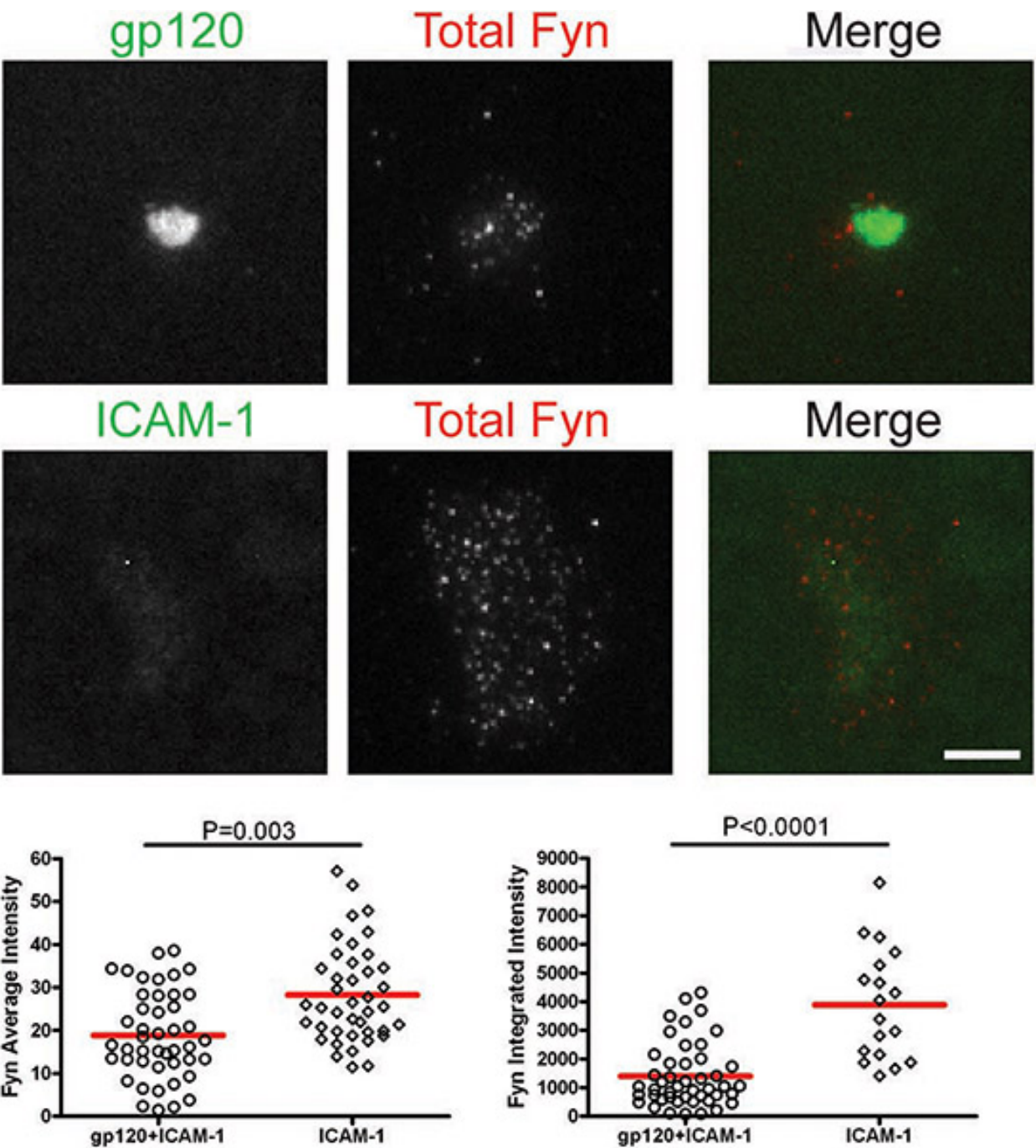

Figure 3. $\mathrm{CD}^{+} \mathrm{T}$ cells were introduced onto bilayers containing gp120 and ICAM-1 or ICAM-1 alone for 45 min and then fixed and stained for total Fyn.

\section{Discussion}

Previous studies have visualized VS in the cell-cell conjugate system; however these studies did not provide images of high enough resolution to visualize the supramolecular structures at the synapse. In our lab, we utilized the glass-supported planar bilayer system to represent the surface of infected cells expressing the virus envelope gp120 and the cellular adhesion molecule ICAM-1. In conjunction with TIRF microscopy, which detects fluorescence signals within 100-200 nm from the bilayer surface with a high signal-to-noise ratio, we were able to detect supramolecular segregation of gp120 from ICAM-1 at the VS. Moreover, the standard immunostaining method can be applied to the bilayer system and was used here to detect and quantify the specific recruitment of active Lck, but not Fyn, to the gp120-contact area at VS ${ }^{15}$. Hence, the planar bilayer offers an experimental system for high-resolution imaging of synapse interface in a 2D plane by TIRF microscopy, as well as wide-field or confocal illumination methods. However, the system also has limitations as adjusting ligand mobility, out-of-plane bending, and fluctuations of biological membranes are not reproduced by planar bilayers. Furthermore, this is an in-vitro system and therefore has other limitations, such as lack of other membrane molecules that would be present on an infected cell and the cytoskeleton machinery that regulates molecular mobility and cellular motility. Also, the dynamics and distribution of the molecules, such as trimers versus monomers of gp120, may not be represented physiologically on the bilayer. Nevertheless, even with these limitations, this system is still very valuable for studying virus-cell or cell-cell interactions, and these methods can serve as a useful guide to researchers who are seeking high-resolution images to detect supramolecular organization that is not discernible in the conventional cell-cell conjugate system.

\section{Disclosures}

The authors declare no conflict of interests. 


\section{Acknowledgements}

This work was supported by NIH grants AI071815 (C.E.H.) and the Roadmap Nanomedicine Development Center award PN2EY016586 (M.L.D.).

\section{References}

1. Bastiani, L., Laal, S., Kim, M., \& Zolla-Pazner, S. Host cell-dependent alterations in envelope components of human immunodeficiency virus type 1 virions. J. Virol. 71, 3444-3450 (1997).

2. Dimitrov, D.S., Willey, R.L., Sato, H., Chang, L.J., Blumenthal, R., \& Martin, M.A. Quantitation of human immunodeficiency virus type 1 infection kinetics. J. Virol. 67, 2182-2190 (1993).

3. Dustin, M.L. Adhesive Bond Dynamics in Contacts between T Lymphocytes and Glass-supported Planar Bilayers Reconstituted with the Immunoglobulin-related Adhesion Molecule CD58. J. Biol. Chem. 272 (25), 15782-15788 (1997).

4. Fortin, J.F., Cantin, R., Lamontagne, G., \& Tremblay, M. Host-derived ICAM-1 glycoproteins incorporated on human immunodeficiency virus type 1 are biologically active and enhance viral infectivity. J. Virol. 71, 3588-3596 (1997).

5. Frank, I., Stoiber, H., Godar, S., Stockinger, H., Steindl, F., Katinger, H.W., \& Dierich, M.P. Acquisition of host cell-surface-derived molecules by HIV-1. Aids. 10, 1611-1620 (1996).

6. Hioe, C.E., Bastiani, L., Hildreth, J.E., \& Zolla-Pazner, S. Role of cellular adhesion molecules in HIV type 1 infection and their impact on virus neutralization. AIDS Res. Hum. Retroviruses. 14 Suppl 3, S247-S254 (1998).

7. Hioe, C.E., Chien, P.C., Jr., Lu, C., Springer, T.A., Wang, X.H., Bandres, J., \& Tuen, M. LFA-1 expression on target cells promotes human immunodeficiency virus type 1 infection and transmission. J. Virol. 75, 1077-1082 (2001).

8. Jolly, C., Kashefi, K., Hollinshead, M., \& Sattentau, Q.J. HIV-1 cell to cell transfer across an Env-induced, actin-dependent synapse. J. Exp. Med. 199, 283-293 (2004).

9. Jolly, C., Mitar, I., \& Sattentau, Q.J. Requirement for an intact T-cell actin and tubulin cytoskeleton for efficient assembly and spread of human immunodeficiency virus type 1. J. Virol. 81, 5547-5560 (2007).

10. McDonald, D., Wu, L., Bohks, S.M., KewalRamani, V.N., Unutmaz, D., \& Hope, T.J. Recruitment of HIV and its receptors to dendritic cell-T cell junctions. Science. 300, 1295-1297 (2003).

11. Pearce-Pratt, R., Malamud, D., \& Phillips, D.M. Role of the cytoskeleton in cell-to-cell transmission of human immunodeficiency virus. J. Virol. 682898-2905 (1994).

12. Rasband, W.S. Image J. U.S. National Institutes of Health, Bethesda, Maryland, USA, http://imagej.nih.gov/ij/, (1997-2011).

13. Rizzuto, C.D. \& Sodroski, J.G. Contribution of virion ICAM-1 to human immunodeficiency virus infectivity and sensitivity to neutralization. J. Virol. 71, 4847-4851 (1997).

14. Vardhana, S. \& Dustin, M. Supported Planar Bilayers for the Formation of Study of Immunological Synapses and Kinapse. J. Vis. Exp. (19), e947, DOI: 10.3791/947 (2008).

15. Vasiliver-Shamis, G., Cho, M.W., Hioe, C.E., \& Dustin, M.L. Human immunodeficiency virus type 1 envelope gp120-induced partial T-cell receptor signaling creates an F-actin-depleted zone in the virological synapse. J. Virol. 83, 11341-11355 (2009).

16. Vasiliver-Shamis, G., Tuen, M., Wu, T., Starr, T., Cameron, T., Thomson, R., Kaur, G., Liu, J., Visciano, M., Li, H., Kumar, R., Ansari, R., Han, D., Cho, M., Dustin, M.L., \& Hioe, C.E. Human immunodeficiency virus type 1 envelope gp120 induces a stop signal and virological synapse formation in noninfected CD4+ T cells. J. Virol. 82 (19), 9445-57 (2008).

17. Zhu, P., Liu, J., Bess, J., Chertova, E., Lifson, J., Grise, H., Ofek, G., Taylor, K., \& Roux, K. Distribution and three-dimensional structure of AIDS virus envelope spikes. Nature. 441, 847-852 (2006). 